\title{
Analysis of the terrestrial locomotion of a salamander robot
}

\author{
Konstantinos Karakasiliotis and Auke Jan Ijspeert
}

\begin{abstract}
Salamanders propel themselves by proper coordination of limb movements and body undulations. This type of locomotion is interesting for robotics to design robots capable of locomotion on water and land. In this work we identify the control and structural parameters that contribute to forward terrestrial locomotion. We introduce a kinematic model of Salamandra robotica II, a new salamander robot, to explore how the stride length varies with different limb sizes and different types of body oscillations. We also perform systematic tests using a dynamic model built in a physics-based simulator to analyze the locomotion performance in terms of forward speed and power consumption. The results show that it is beneficial to use body undulations with variable curvature along the body, and that the tail can serve as a fifth limb to provide thrust on ground. Experiments using the real robot validate the simulation results and the contribution of the proposed control strategies.
\end{abstract}

\section{INTRODUCTION}

"Vertebrate morphologists have a long history of using salamanders as a window into the locomotor capabilities of primitive tetrapods". With this phrase, Ashley-Ross [1], p. 225, reveals the great interest of biologists in studying the salamander's locomotor mechanisms. This interest does not lie only in the understanding of the biological control mechanisms at the neuronal level [2]-[4], but also, on the locomotor morphology and kinematics [1], [5]-[9]. However, little interest has been shown from roboticists in this direction and previous studies mainly focus on biologically inspired control mechanisms [10]-[13] aiming at addressing questions about the neuromechanical structure and its interaction with the body. To the best of our knowledge, no other detailed study on mechanical salamander kinematics and structural characteristics has been published yet in the robotics literature. Studying salamander, as well as lizard and crocodilian, kinematics separately from other tetrapod categories seems indispensable. They differ from most of the others by still retaining several features of the primitive tetrapod locomotion related to their sprawling posture. The basic characteristics of salamander locomotion are based on the coordination of limb movements and body waves, producing, generally, symmetric gaits.

In previous work [10], carried out at our lab, a salamander robot was presented, capable of terrestrial and aquatic locomotion. In this paper, we present the second generation of the salamander robot, Salamandra robotica II (Fig. 1). Based on the general structural characteristics of this robot we focus on the study of its kinematics and we try to analyze the

Both authors are with the the Biologically Inspired Robotics Group, Swiss Federal Institute of Technology (EPFL).

This work is part of the LAMPETRA project funded by the Seventh EU framework programme under the theme ICT-2007.8.3-FET proactive 3.

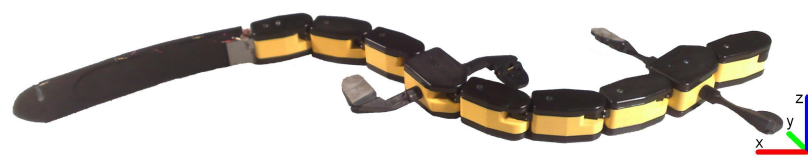

Fig. 1. Salamandra robotica II

impact of several of these characteristics on the locomotion performance. The body of the robot consists of 9 segments serially connected by 8 joints. Four of these joints, located between the limbed segments, represent what we call the trunk. Three joints are used for the tail and the last one is dedicated to the head. The axis of rotation of all the joints are parallel, thus, the body of the robot can undulate only in the horizontal plane. The limbs, similarly to the double crank mechanism proposed by Barclay [14], use a fixed knee joint and are able to rotate around the lateral axis, perpendicular to both the body axis and the body joint rotation axis.

The paper is organized as follows. In the first section we describe the kinematic model of the salamander robot and we investigate various parameters that affect the kinematic behavior during terrestrial locomotion. We also describe the control methods which were used during this investigation. In the second section we present a dynamic simulation model of the salamander robot and we investigate structural characteristics and control strategies related to spine undulations of variable curvature. We systematically evaluate the locomotion performance in terms of speed and power consumption and we discuss the results of this investigation. Finally, we perform similar experiments with the real robot and evaluate our initial hypotheses.

\section{MODELING SALAMANDER LOCOMOTION}

\section{A. Kinematic model}

In order to understand the locomotor behavior of the salamander's mechanical model, we introduce a simplified kinematic model and we exclude the body dynamics. The purpose of the model is to explore how kinematic considerations affect the stride length as well as possible slipping of the feet. For instance, the head and tail chains can be neglected since they do not affect the terrestrial locomotion patterns if dynamics are excluded. However, we will see their role in the presence of dynamics in later sections. Further simplification is feasible due to symmetries, both in body structure and gaits. As a result, the remaining chains are the ones which describe the trunk and two contralateral limbs, e.g. the left hind limb and the right forelimb. 


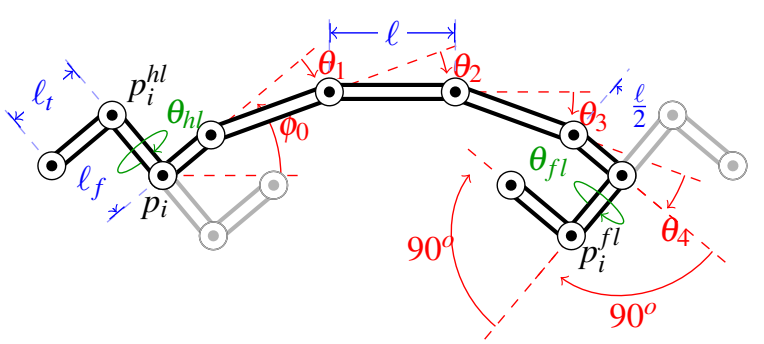

Fig. 2. The kinematic model of the salamander robot (top view). The circles represent joints and endpoints and the double lines the body and limb segments. The body joint angles are denoted by $\theta_{i}$ and the body segment length by $\ell$ and $\ell / 2$. The limb rotations are denoted by $\theta_{h l}$ and $\theta_{f l}$ for the hind and forelimb respectively, and their direction by green arrows. The limb rotation axis is fixed to the normal lateral direction and the knee angle is also fixed at $90^{\circ}$.

In Fig. 2 the body and limb segments are represented by two parallel lines and the joints and endpoints by circles. The central curved linkage represents the trunk. Each body joint angle is denoted as $\theta_{i}, 1 \leq i \leq 4$ and the length of each body segment is denoted as $\ell$. Note that the first and last trunk segments have $\ell / 2$ length following the placement of the limbs. The body joint coordinates are denoted as $p_{i}=$ $\left(x_{i}, y_{i}, z_{i}\right), 1 \leq i \leq 6$ and the relative angle of the trunk with the world coordinate system is denoted as $\phi_{0}$. The rotations of the limbs are denoted as $\theta_{h l}$ and $\theta_{f l}$ for the hind and forelimb respectively. The femur length is denoted as $\ell_{f}$ and the tibia length as $\ell_{t}$. All the limbs are considered to have the same size. The limb coordinates are denoted as $p_{i}^{h l}=$ $\left(x_{i}^{h l}, y_{i}^{h l}, z_{i}^{h l}\right), 1 \leq i \leq 2$ and $p_{i}^{f l}=\left(x_{i}^{f l}, y_{i}^{f l}, z_{i}^{f l}\right), 1 \leq i \leq 2$ for the hind and forelimb respectively.

The coordinates of the trunk are given by

$$
p_{i}^{T}=\left(\begin{array}{c}
\sum_{i=2}^{6} \ell_{i-1} \cos \left(\phi_{0}-\sum_{j=1}^{i-1} \theta_{j}\right) \\
\sum_{i=2}^{6} \ell_{i-1} \sin \left(\phi_{0}-\sum_{j=1}^{i-1} \theta_{j}\right) \\
h_{s}
\end{array}\right), p_{1}^{T}=\left(\begin{array}{l}
0 \\
0 \\
0
\end{array}\right),
$$

where $h_{s}$ is the height of the robot's hip joints.

The coordinates of the hind limb end point are given by

$$
p_{2}^{h l^{T}}=\left(\begin{array}{c}
-\cos \left(\phi_{0}\right) \cos \left(\theta_{h l}\right) \ell_{t}-\sin \left(\phi_{0}\right) \ell_{f} \\
-\sin \left(\phi_{0}\right) \cos \left(\theta_{h l}\right) \ell_{t}+\cos \left(\phi_{0}\right) \ell_{f} \\
\sin \left(\theta_{h l}\right) \ell_{t}+h_{s}
\end{array}\right) .
$$

We derive the forelimb's end point $p_{2}^{f l}$ in a similar way.

\section{B. Forward walking}

Salamanders are known to use mainly standing waves during walking [1], [6]. To model this, a simple sine controller determines the body joint angles which are given by

$$
\theta_{i}(t)=A \sin (2 \pi f t), 1 \leq i \leq 4
$$

where $A$ is the body wave's amplitude and $f$ its frequency.

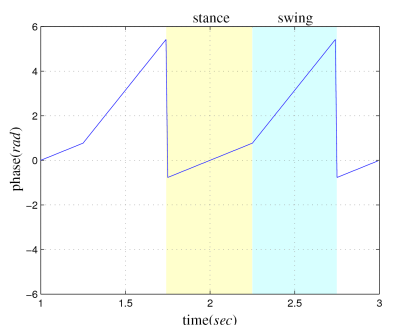

(a)

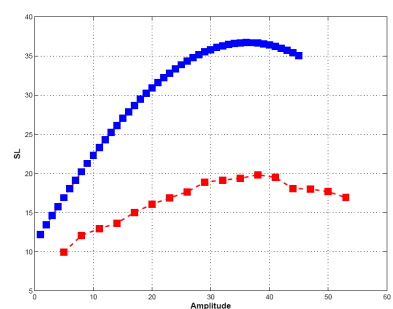

(b)
Fig. 3. (a) Hind and forelimb rotations during stance and swing phases (yellow and cyan regions respectively). (b) The stride length as a function of body wave amplitude for the kinematic model (blue) and the real robot (red).

In order to take the maximum advantage of the body undulation for the stride length, we should synchronize the maximum body curvature on the right side with the touchground event of the right forelimb and the maximum body curvature on the left side with the lift-off of the same limb. To do so, we divide the limb rotation in two phases, the stance and the swing. Since the axis of the limbs' rotation is placed at a height $h_{s}$ these two phases have different durations if the limb's rotational speed is constant. Different rotation speeds must be applied in order to achieve the same durations for both phases (Fig. 3(a)). Eq. 4 gives the rotation of the hind limb with respect to the time.

$$
\theta_{h l}(t)= \begin{cases}4 \theta_{h l}^{T G} f t & \text { if } 0 \leq t<\frac{1}{4} \\ 4\left(\pi-\theta_{h l}^{T G}\right) f t+2 \theta_{h l}^{T G}-\pi & \text { if } \frac{1}{4} \leq t<\frac{3}{4} \\ 4 \theta_{h l}^{T G} f(t-1) & \text { if } \frac{3}{4} \leq t<1\end{cases}
$$

where $\theta_{h l}^{T G}$ is the angle at which the limb touches the ground.

Applying the above control for a body wave amplitude of $A=30^{\circ}$ we derive the trajectories drawn in Fig. 4(b). The blue lines correspond to the trajectories of the feet during stance phase and the red lines to the trajectories of the center of mass of each body segment. The difference found in the curvature of the feet trajectories yields a variable distance between the feet on the ground during stance. A too large variable distance is to be avoided since it either leads to high internal torques (if the feet do not slide) or to slipping with major impact on the stride length. This effect increases with respect to the body wave amplitude. Fig. 4(a) shows the sliding distance as a function of amplitude. The red line corresponds to the sliding only on the $x$-axis and the blue on the $x y$-plane.

The stride length corresponds to the distance traveled during the time from the touch-ground of the hind limb to the next touch-ground of the same. Thus, given that the hind foot moves symmetrically around $y$-axis and taking into account both hind limbs, the stride length is given by

$$
S_{L}=4 x_{1}^{T G},
$$

where $x_{1}^{T G}$ is the value of the $x$ coordinate of the hind foot when it touches the ground. 


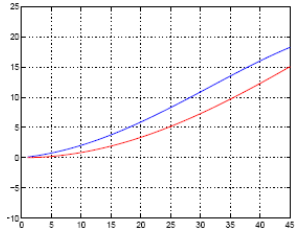

(a)

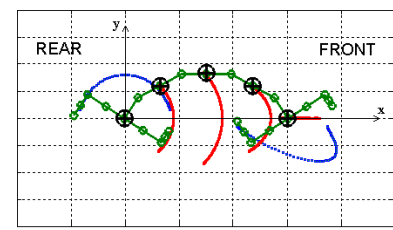

(b)
Fig. 4. (a) Sliding (cm) at $x$-axis (red) and at both axes (blue) as a function of the body amplitude. (b) The trajectories of the feet (blue) and segments' center of mass (red) during half of a stride.

Experiments with the kinematic model and the real robot for the stride length as a function of body wave amplitude show that there is a maximum value of amplitude where the stride length starts to decay (Fig. 3(b)). The quantitative difference between the model and the robot is expected since in the kinematic model we assume that the hind foot is anchored on the ground, while it often slips with the real robot.

Different types of legs can be attached to the robot. In order to guide the design of the legs we carried out a systematic search for the impact of the limb femur and tibia lengths on the forward speed for various amplitudes. The results are presented in Fig. 5. The linear relation between both lengths and the resulting speed does not provide enough information in order to converge to an optimal choice. Only two limitations arise for both limb lengths. The femur length is limited by the internal collisions at high amplitudes. The tibia length should be kept near the height of the limb rotation axis $\left(h_{s}\right)$ in order to avoid high lifting of the robot at each step and, thus, hardware damages due to high-impact collisions with the ground. However, the speed for any given tibia length $\left(\ell_{t}\right)$ is decreasing as the amplitude $(A)$ increases. This could suggest that the final decision could depend mostly on the femur length $\left(\ell_{f}\right)$ which increases appreciably with respect to the body wave amplitude. As a compromise, we therefore decided to design new legs with femurs of $13 \mathrm{~cm}$ and tibias of $4.5 \mathrm{~cm}$.

\section{EXPLORING SALAMANDER LOCOMOTION}

\section{A. Motivation}

In this section we explore some features of the salamander locomotion which involve both structural and control parameters.

An interesting observation from the INSERM neurobiologist Jean-Marie Cabelguen of the University of Bordeaux, France, shows the significant change of the tail functionality during forward stepping on a slippery surface (Fig. 6). We hypothesise that when the animal senses that its feet are slipping it rapidly waves the tail at very high amplitudes in order to increase the total contacting surface on the substrate yielding higher thrust. The tail can then be seen as a fifth limb. This could make a useful feature for the mechanical salamander which suggests further exploration.

Moreover, although the double crank mechanism proposed by Barclay works fairly well, as shown by the kinematic

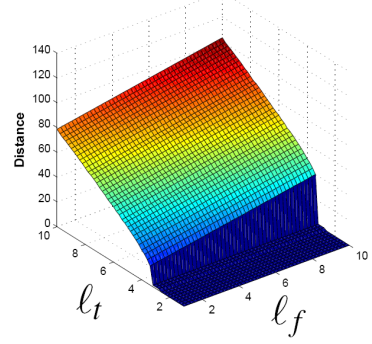

(a) $A=10^{\circ}$

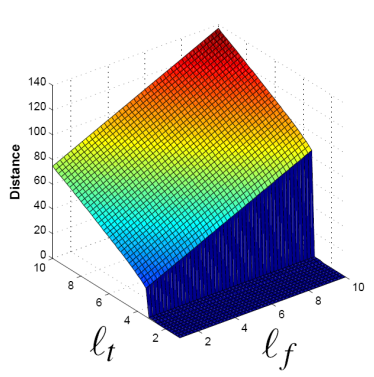

(c) $A=30^{\circ}$

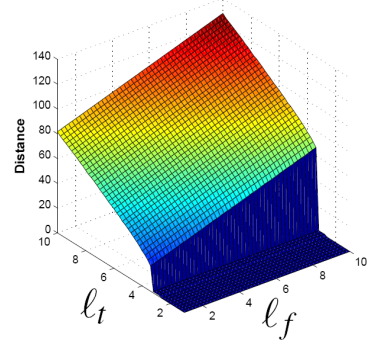

(b) $A=20^{\circ}$

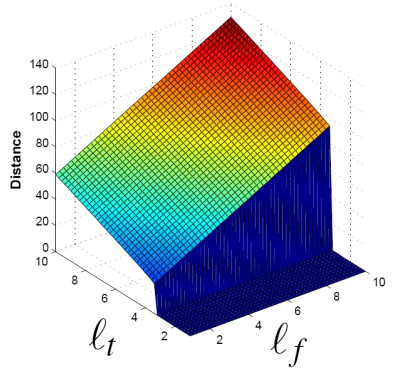

(d) $A=40^{\circ}$
Fig. 5. Distance traveled $(\mathrm{cm})$ during two strides as a function of the limb femur and tibia lengths $(\mathrm{cm})$.

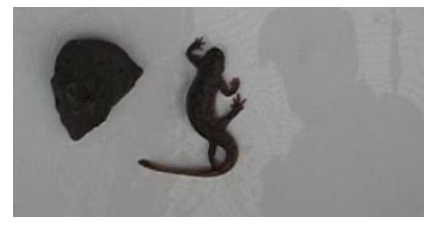

Fig. 6. Snapshot of the salamander using the tail in very high amplitude to avoid slipping during walking on a slippery surface. (Jean-Marie Cabelguen, University of Bordeaux, France).

model, kinematic data from a real salamander show that the angle between the pelvic girdle and the femur varies, increasing the stride length. Driven by that, Ashley-Ross [1], pp. 274, proposed a more realistic model of the hind limb which actuates the hip joint, not as a rotational degree of freedom, but as an elliptical one. Even though it is not possible to directly incorporate this feature to our robot, since hip joint elliptical actuation adds further complexity to the hardware design, it can provide inspiration for different control approaches as we will see in the next sections.

\section{B. Simulated salamander robot model}

In order to investigate the tail use and the possible elliptical actuation of the hip joint according to their effect on the mechanical salamander we introduced a model (Fig. 7) developed in the mobile robot simulator Webots, from Cyberbotics. Following the basic characteristics of the real robot (Fig. 1) the model consists of eight joints able to rotate along the $z$-axis and of four limbs with rigid knees at $90^{\circ}$ able to rotate around the $y$-axis. The $x$-axis is supposed tangential to the body. To better match the model with the real robot we simulated a spring and damper rotational joint with stops around the $y$-axis between consecutive segments which illustrates the vertical bending of the robot due to 


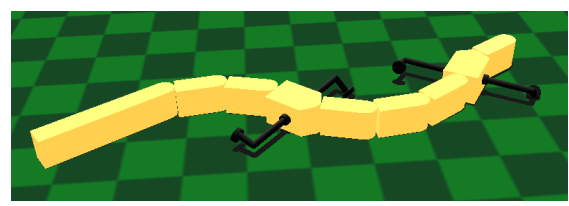

Fig. 7. The simulated Salamandra robotica II with a long tail and increased femur lengths compared to Salamandra robotica I [10].

the elasticity of the mechanical connections. The mass was equally distributed in each body segment ( $220 \mathrm{~g}$ for each) apart from the limb segments which weighted $390 \mathrm{~g}$. Frictional measurements with the robot on a wooden floor determined the Coulomb friction coefficient of the simulator at the value of $\mu=0.25$. The femur and tibia lengths, as in the real robot, were $13 \mathrm{~cm}$ and $4.5 \mathrm{~cm}$ respectively.

\section{Experiments}

In the following experiments of this section we will see how the new features driven by the kinematic model and our motivation from the real animal affect the locomotion performance both in simulation and with the real robot.

Coming back to our second motivation which refers to the variation of the pelvic girdle-femur angle, we introduce a different control approach which imposes a standing wave with a curvature that varies along the spine, in other words with a different amplitude of oscillation at each joint. In this way, if higher amplitudes are applied on the limb segments with respect to the rest of the spine, the resulting posture gets closer to the one which uses an elliptical hip joint. An example of such a posture is given in Fig. 8. Extending this idea to the tail part we can control the extent of tail use. As a result, we introduce a general control approach with three groups of amplitudes: one for the limb segment joints, one for the trunk joints and one for the tail joints (Fig. 9). The amplitudes of the same group have the same value.

Using these three amplitude groups as open parameters and by systematically testing their effect on the locomotion performance we can derive useful information about the role of the tail and the standing wave postures. The range of each of these amplitudes was chosen after manual search

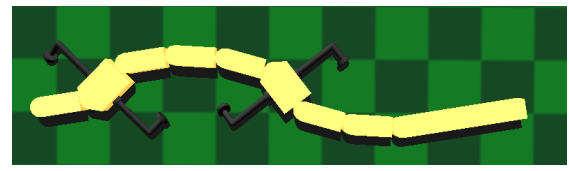

Fig. 8. Posture of the standing wave when higher amplitudes are applied to the limb segments. Notice how this higher bend resembles a possible movement of a pelvic girdle-femur angle (elliptical hip activation).

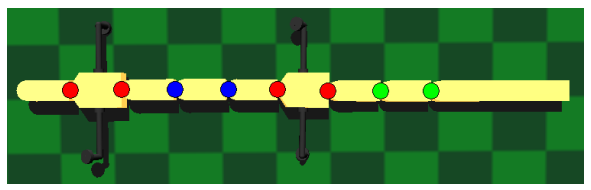

Fig. 9. Standing wave with variable curvature approach. (red) Limb segment amplitude, (blue) Trunk amplitude, (green) Tail amplitude. of the interesting and collision free regions. In simulation we investigate trunk amplitudes from $0^{\circ}$ to $25^{\circ}$ with a step of $5^{\circ}$, limb segment amplitudes from $10^{\circ}$ to $40^{\circ}$ with the same step and tail amplitudes from $0^{\circ}$ to $75^{\circ}$ with a step of $25^{\circ}$. We performed the same series of experiments for different frequencies starting from $0.3 \mathrm{~Hz}$ and stopping at $1.8 \mathrm{~Hz}$, where the motors start to be unable to follow the given postures, with a step of $0.3 \mathrm{~Hz}$. However, in this paper we will discuss, as an example, a middle frequency of $1.2 \mathrm{~Hz}$.

In the real robot experiments the range of trunk amplitudes varied from $0^{\circ}$ to $25^{\circ}$ with a step of $12.5^{\circ}$, the limb segment amplitudes from $10^{\circ}$ to $40^{\circ}$ with $10^{\circ}$ step and tail amplitudes from $0^{\circ}$ to $50^{\circ}$ with a step of $25^{\circ}$. The frequency used for these experiments was $0.5 \mathrm{~Hz}$ to avoid high mechanical stress.

\section{Results and discussion}

1) Simulation: Fig. 10 shows an example of speed measurement results at $1.2 \mathrm{~Hz}$, which is a middle frequency, for different trunk, tail and limb segment amplitudes. Each contour plot is dedicated to a different tail amplitude from $0^{\circ}$ to $75^{\circ}$. On the $y$-axis the limb segment amplitude is increasing downwards while on the $x$-axis the trunk amplitude is increasing towards the right.

Before getting into the quantitative details, already some very interesting observations can be made from a more qualitative view. From the first (Fig. 10(a)) to the last plot (Fig. 10(d)), it seems that there is a movement of the lighter region from the lower left to the lower right and that its area and intensity are growing. On this direction the tail amplitude is increasing. In addition, the lighter the region gets, the higher the speed. These results indicate that an additional thrust is obtained from the increased tail movements. The lighter region (higher speed) is constantly at the lower part of the plots, where the difference between the trunk and limb segment amplitudes is higher. This proves the effectiveness of the proposed control approach based on variable body curvature. In the kinematic analysis we suggested that the stride length increases, up to a limit, with respect to the body curvature. This does not seem to be true in the Fig. 10(a) and (b). Feet slipping, due to the body undulations, as we described in Fig. 4, and due to the frictional properties between the feet and the ground, seems to be a critical phenomenon when dynamics are considered. However, as the tail amplitude increases, this effect decays. The performance enhancement with the use of the tail is validated by simulations of the same robot but without the tail segments (Fig. 13(a)).

Fig. 11 and Fig. 13(b) show the results for the power consumption of the tailed and tailless robot, respectively, for the same parameters at the same frequency as before. To measure the power consumption each motor was modeled as an RL circuit in series with a counter-electromotive force. In the calculations we considered the characteristic values of the Faulhaber 1724003 SR motor, used at each joint. In order to better visualize our data, the value ranges between tailed and tailless robot are different since the value range of 


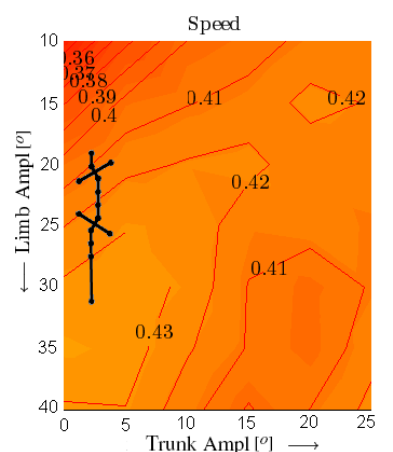

(a) Tail amplitude $=0^{\circ}$

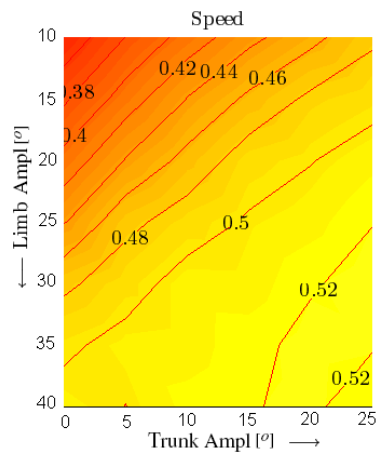

(c) Tail amplitude $=50^{\circ}$

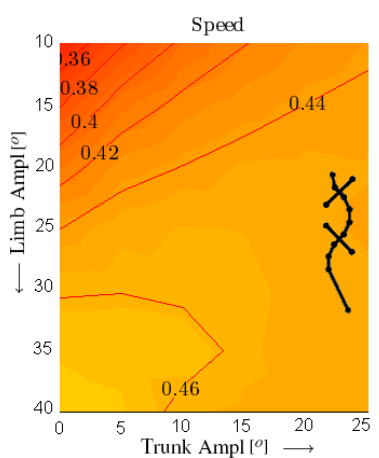

(b) Tail amplitude $=25^{\circ}$

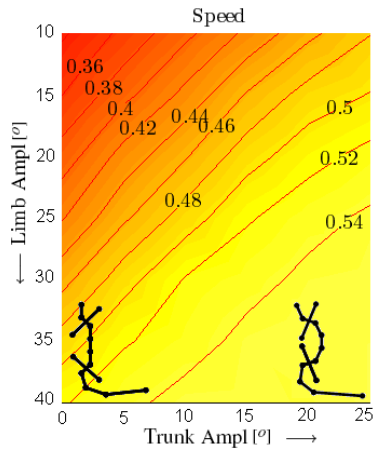

(d) Tail amplitude $=75^{\circ}$

Fig. 10. Simulation: The speed $(\mathrm{m} / \mathrm{s})$ as a function of the trunk and the limb segment amplitudes for a tail amplitude of (a) $0^{\circ}$, (b) $25^{\circ}$, (c) $50^{\circ}$ and (d) $75^{\circ}$. Red represents lower values of speed and yellow higher. The leg segment amplitude is increasing downwards on the $y$-axis and the trunk amplitude towards the right on the $x$-axis. The frequency for this example is $1.2 \mathrm{~Hz}$. In (a) and (d) three extreme postures for tail, limb and trunk amplitudes are shown and can be compared to a posture of all amplitudes equal to $25^{\circ}$, shown in (b).

the latter is three times lower. From these plots it is clear that the power consumption is less at the upper left and middle regions which shows that an increment in amplitude for any parameter yields higher consumption (as could be expected). However, the variation for a given value of tail amplitude (each independent plot) is low. This suggests that the tail use affects significantly the power consumption compared to the other amplitudes. To explore that, we introduce a new metric function,

$$
F_{f}=\left(\frac{S-\min (S)}{\max (S)-\min (S)}+\frac{\max (C)-C}{\max (C)-\min (C)}\right) / 2,
$$

where $S$ and $C$ denote the speed and power consumption respectively. $F_{f}$ increases with speed and decreases with power consumption, and therefore gives us a trade-off between speed and energy efficiency. For the experiments of Fig. 10 and Fig. 11 we calculate this new metric (Fig. 12). For the middle and low values of tail amplitude the results seem closer to the results of the speed in Fig. 10. However, for higher tail amplitudes the power consumption starts to affect much more the final trade-off, upper bounding the positive effect of the tail.

2) Real robot: The real robot experiments are shown in Fig. 14. In Fig. 14(a) the tail of the robot was removed while

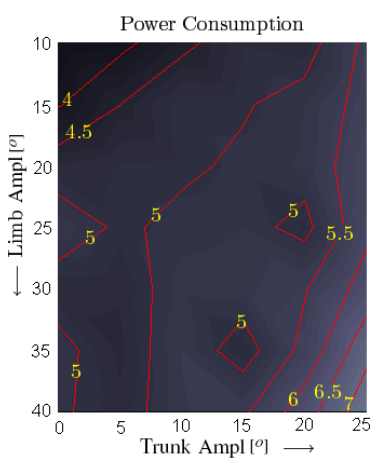

(a) Tail amplitude $=0^{\circ}$

Power Consumption

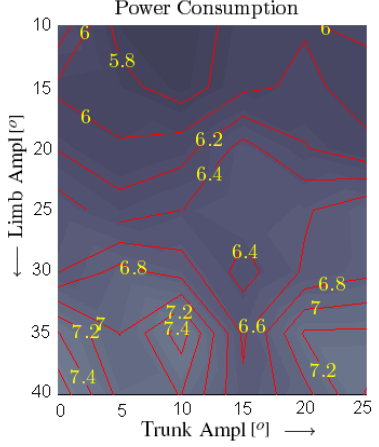

(c) Tail amplitude $=50^{\circ}$

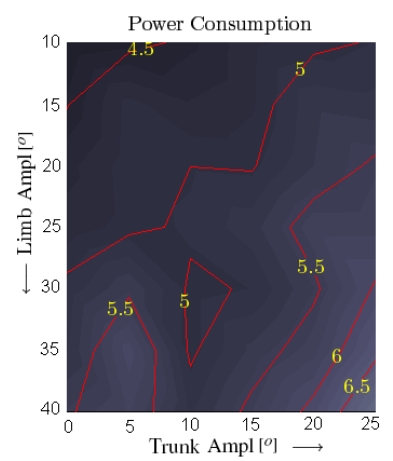

(b) Tail amplitude $=25^{\circ}$

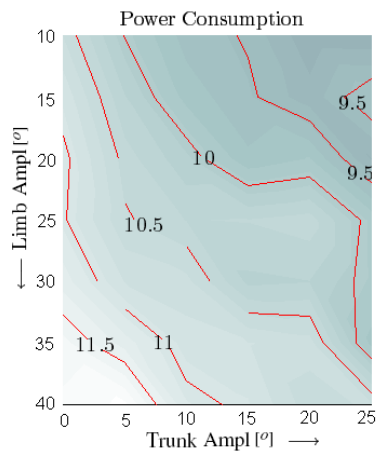

(d) Tail amplitude $=75^{\circ}$
Fig. 11. Simulation: The power consumption (W) as a function of the trunk and the limb segment amplitudes for a tail amplitude of (a) $0^{\circ}$, (b) $25^{\circ}$, (c) $50^{\circ}$ and (d) $75^{\circ}$. The frequency for this example is $1.2 \mathrm{~Hz}$.

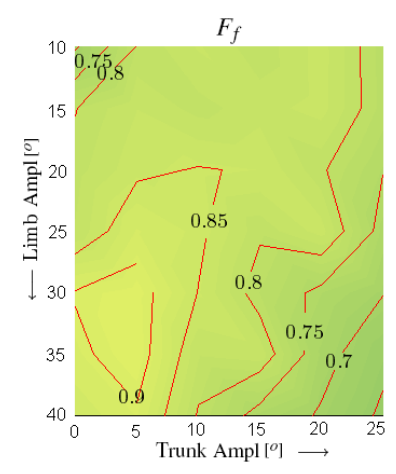

(a) Tail amplitude $=0^{\circ}$

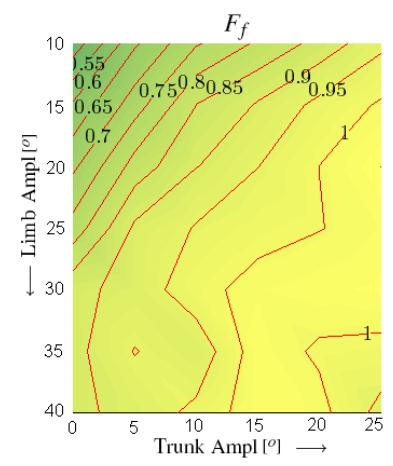

(c) Tail amplitude $=50^{\circ}$

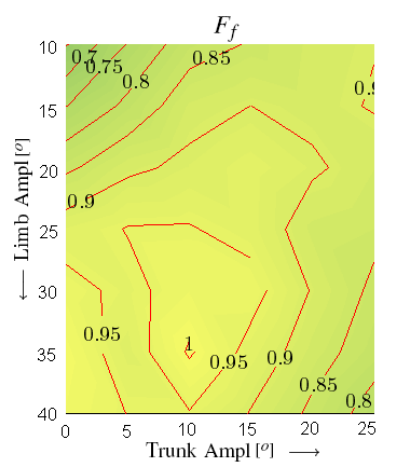

(b) Tail amplitude $=25^{\circ}$

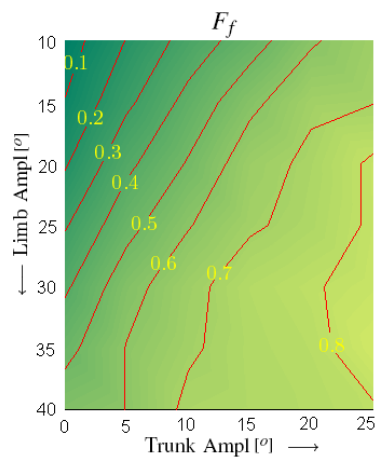

(d) Tail amplitude $=75^{\circ}$
Fig. 12. Simulation: The $F_{f}$ as a function of the trunk and the limb segment amplitudes for a tail amplitude of (a) $0^{\circ}$, (b) $25^{\circ}$, (c) $50^{\circ}$ and (d) $75^{\circ}$. The frequency for this example is $1.2 \mathrm{~Hz}$. 


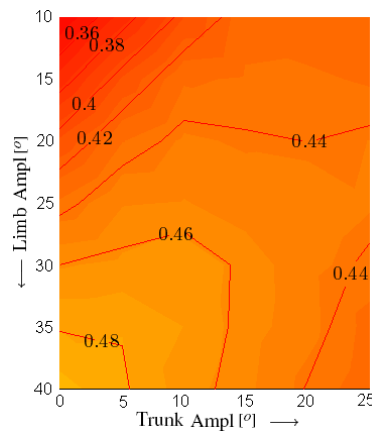

(a) Speed $(\mathrm{m} / \mathrm{s})$

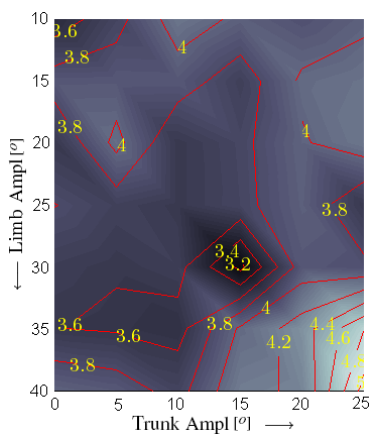

(b) Power consumption (W)
Fig. 13. Simulation: The speed and power consumption of the tailless salamander robot as a function of the trunk and limb segment amplitudes. The frequency for this example is $1.2 \mathrm{~Hz}$.

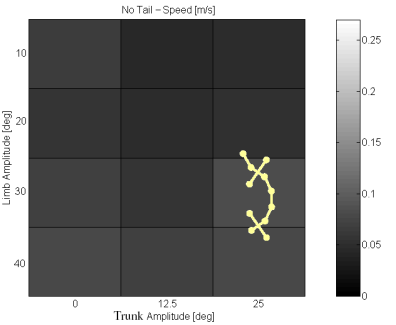

(a) Tailless robot

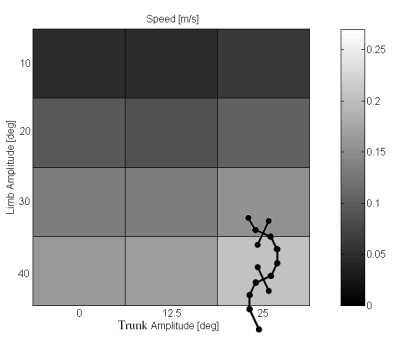

(c) Tail amplitude $=25^{\circ}$

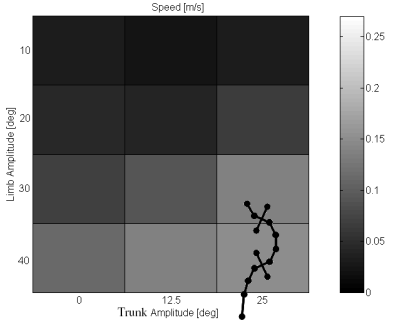

(b) Tail amplitude $=0^{\circ}$

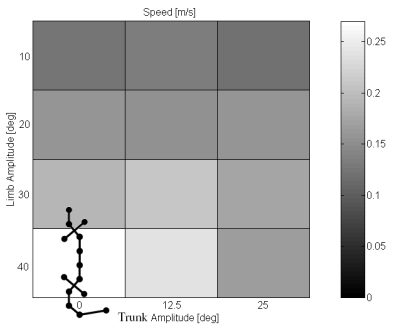

(d) Tail amplitude $=50^{\circ}$
Fig. 14. Real robot: Speed $(\mathrm{m} / \mathrm{s})$ as a function of the trunk and the limb segment amplitudes for (a) the tailless configuration and a tail amplitude of (b) $0^{\circ}$, (c) $25^{\circ}$, (d) $50^{\circ}$. The frequency of these experiments is $0.5 \mathrm{~Hz}$. The posture of the robot for the best parameters is shown at each plot.

in the rest of the plots the amplitude of the tail is changing from $0^{\circ}$ to $50^{\circ}$. In order to extend the last tail segment of the robot as in the simulation, a long piece of rubber was connected to its end (Fig. 1). Due to hardware problems, the power consumption of the real robot is not included in the present paper, but it will be measured in our future work.

In the tailless configuration (Fig. 14(a)) the speed is almost evenly distributed with its maximum values $(\sim 0.08 \mathrm{~m} / \mathrm{s})$ lying at high limb segment amplitudes combined with high trunk amplitudes (bottom-right). In Fig. 14(b) the disuse of the long tail yields worse results at low amplitudes compared to the tailless configuration. The long tail configuration gradually overcomes the tailless one as higher amplitudes are used for both limb and trunk groups. In the same way in Fig. 14(c) the highest values of speed are found at the bottom-right. This gradual increment of the speed could be explained by the fact that the tail weight increases the normal force applied on the hind limbs therefore decreasing the feet slipping. However, the major difference between the Fig. 14(b), (c) and (d) is the speed increment (up to $\sim 0.27$ $\mathrm{m} / \mathrm{s}$ ) which validates, also in the real robot, that the use of the tail and in general standing waves with variable amplitudes along the spine can enhance the locomotion performance.

\section{CONCLUSION}

Starting from a simplified kinematic model of the salamander robot we mathematically expressed the body and limb coordination and we suggested that the body amplitude and femur lengths affect significantly the stride length. Inspired by real salamanders we introduced two hypotheses: that standing waves with variable amplitudes along the spine and more specifically high tail amplitudes contribute to the forward thrust. Experiments in simulation and with the real robot validated these two hypotheses.

\section{ACKNOWLEDGMENTS}

The authors gratefully acknowledge Jean-Marie Cabelguen for providing valuable information on salamanders, Jeremie Knuesel for the formulation of power consumption, and Robert J. Full, Justin Seipel, and Ardian Jusufi for useful discussions on the biomechanics of salamander locomotion.

\section{REFERENCES}

[1] M. A. Ashley-Ross, "Hind limb kinematics during terrestrial locomotion in a salamander (Dicamptodon Tenebrosus)", J. exp. Biol., 1994, 193, pp. 255-283.

[2] J.M. Cabelguen, C. Bourcier-Lucas and R. Dubuc, "Bimodal Locomotion Elicited by Electrical Stimulation of the Midbrain in the Salamander Notophthalmus viridescens", The Journal of Neuroscience, 2003, March 15, 23(6):2434.

[3] I. Delvolve, P. Branghereau, R. Dubuc, and J.M. Cabelguen, "Fictive Rhythmic Motor Patterns Induced by NMDA in an In Vitro Brain Stem-Spinal Cord Preparation From an Adult Urodele", J. Neurophysiol., 1999, 82: 1074-1077.

[4] G. Szekely, G. Czeh and G. Voros, "The activity pattern of limb muscles in freely moving normal and deafferented newts", Expl Brain Res., 1969, 9, 5362.

[5] A. B. Howell, "Speed in Animals", New York: Hafner Publishing Company, 1944.

[6] J. Gray, "Animal Locomotion", New York: W. W. Norton \& Company, 1968.

[7] M. Hildebrand, "Analysis of the symmetrical gaits of tetrapods", Fol. Biotheor, 1966, 6, 922.

[8] S. C. Rewcastle, "Stance and gait in tetrapods: an evolutionary scenario", Symp. zool. Soc. Lond., 1981, 48, 239267.

[9] C. Gans and G. de Gueldre, "Striated muscle: physiology and functional morphology", In Environmental Physiology of the Amphibians (ed. M. E. Feder and W. W. Burggren), Chicago, IL: University of Chicago Press, 1992, pp. 277313.

[10] A.J. Ijspeert, A. Crespi, D. Ryczko, and J.M. Cabelguen, "From swimming to walking with a salamander robot driven by a spinal cord model", Science, 9 March 2007, Vol. 315. no. 5817, pp. $1416-1420$.

[11] A.J. Ijspeert, A. Crespi, and J.M. Cabelguen, "Simulation and robotics studies of salamander locomotion. Applying neurobiological principles to the control of locomotion in robots", Neuroinformatics, 2005, 3(3):171-196.

[12] A.J. Ijspeert, "A connectionist central pattern generator for the aquatic and terrestrial gaits of a simulated salamander", Biological Cybernetics, 2001, 84(5):331-348.

[13] P. Manoonpong, F. Pasemann, J. Fischer, H. Roth, "Neural processing of auditory signals and modular neural control for sound tropism of walking machines", International Journal of Advanced Robotic Systems ,2005 , pp. 223234.

[14] O. R. Barclay, "The mechanics of amphibian locomotion", J. exp. Biol., 1946, 23, pp. 177203. 